\title{
Innere Archäologien des Positivismus?
}

\section{Eine Bestandsaufnahme zu neueren Übersetzungen aus dem Feld der Historischen Epistemologie}

Thomas Ebke

Die Verfestigung der Ausdrücke „Wissenschaftstheorie“, „Wissenschaftsphilosophie“ und „Wissenschaftsgeschichte“ zu veritablen termini technici ist eine für die europäische Philosophie des 19. Jahrhunderts überaus denkwürdige Entwicklung. Etwa um 1840 herum lässt sich für den frankophonen, den angelsächsischen und den deutschsprachigen Raum eine gewisse Autonomisierung der drei genannten Konzepte (in ihrer nationalsprachlich je spezifischen Notation) konstatieren: Fortan korrelieren „Wissenschaftstheorie" (théorie de la science oder theory of science), "Wissenschaftsphilosophie" (philosophie des sciences oder philosophy of science) und „Wissenschaftsgeschichte" (histoire des sciences oder history of science) jeweils nicht länger als epistemologische Klärungen mit bestimmten Einzelwissenschaften. Während beispielsweise noch Jean d'Alembert und Denis Diderot in der Encyclopédie für jede Einzelwissenschaft und jede für sich stehende „Kunst“ eine der jeweiligen Disziplin korrelativ entsprechende Philosophie vorsahen (Diderot and d'Alembert 1765), deren Rolle es war, den Spezialdisziplinen „Gründewissen bereitzustellen“ (Pulte 2004: 974), ist 1840 am musterhaften Fall von William Whewells dreiteiliger Philosophy of the Inductive Sciences bereits die Vereinheitlichung des Projekts einer „philosophy of science“ zu einer Form der Reflexion ablesbar, die es mit der Befragung der inneren Voraussetzungen und der Verfahrensweisen wissenschaftlichen Wissens überhaupt zu tun hat: „The Philosophy of Science [...] would imply nothing less than a complete insight into the essence and conditions of all real knowledge, and an exposition of the best methods for the discovery of new truths" (Whewell [1840] 1967: 1). 
Wie Helmut Pulte hervorgehoben hat, stellte Whewells Konzeption in dem Maße die Weichen für die im engeren Sinne wissenschaftstheoretischen und wissenschaftshistorischen Diskurse ab der Mitte des 19. Jahrhunderts, wie seine Ausarbeitung einer generalisierten philosophy of science den Naturwissenschaften „bereits als ,historische[n] Formationen', d.h. in ihrer (bes. methodologisch bestimmten) Ausdifferenzierung" Rechnung trug (Pulte 2004: 976). Whewells enge Knüpfung der Wissenschaftstheorie an Wissenschaftsgeschichte sollte in der Tat den Rahmen vorzeichnen, in dem sich unter anderem die erstmalige Präsenz des Rubrums „Wissenschaftstheorie“ im deutschen Kontext (bei Eugen Dühring in Anlehnung an Friedrich Adolf Trendelenburg) sowie die französische Tradition der post-positivistischen critique de la science im Anschluss an Henri Poincaré und Pierre Duhem verorten und begreifen lassen (Pulte 2004: 964 f.).

Drei Phänomene verdienen in diesem veränderten Ensemble der Beziehungen zwischen Wissenschaft, Philosophie und geschichtlicher Reflexion besondere Erwähnung: Erstens ist die betont neue Konzeptualisierung von "Wissenschaft" bzw. „wissenschaftlichem Wissen“ hervorzukehren, die seit dem 18., vor allem aber im Zuge des 19. Jahrhunderts angestammte Wissenschaftsdefinitionen ab- bzw. sogar aufzulösen beginnt. Grundlegende Bedeutung hat in diesem Zusammenhang der Übergang von der Deduktion, die das, was behauptet wird, aus dem zu seiner Behauptung vorausgesetzten und ,vorausgewussten" Allgemeinen folgert, zur Induktion, die im Schritt von den beobachtbaren Einzeltatsachen zu den Gesetzmäßigkeiten eine (relative) Erklärung dieser Partikularitäten ermöglicht. Die mit dieser Umstellung verbundene erkenntnistheoretische Herausforderung der modernen Erfahrungswissenschaften hat, wie die einschlägigen Kontroversen zum Thema des Induktionismus bis hin zu Popper zeigen, einen epistemologisch überaus prekären Charakter. Gleichwohl hängt der Wissenschaftsbegriff fundamental an der Frage nach der Induktion und betrifft die im 19. Jahrhundert proliferierenden empirischen Naturwissenschaften genau so wie den mathematischen Diskurs der philosophischen Logik, mit dem sich die sogenannten exakten Wissenschaften zunehmend zu assimilieren beginnen (vgl. Diemer 1968).

Zweitens führt diese Transformation in der Konzeptualisierung und der Praxis der Wissenschaften, die als in erkenntnistheoretischer Hinsicht problematische Projekte aufgefasst werden, eine Krise der Philosophie als solcher vor. Schon Whewells Redeweise von einer Philosophy of the Inductive Sciences markiert eine Dynamisierung der Rollen von Wissenschaft und Philosophie, die letztere ihrer souveränen metatheoretischen Distanz gegenüber den Einzelwissenschaften enthebt. Von den antiken Auffassungen an über die frühneuzeitlichen Entwürfe bis hin zu Kant und (insbesondere) Hegel hatte sich, mit wechselnden Akzenten, der Anspruch der Philosophie tradiert, höchste Wissenschaft zu sein, also Wissenschaft aller Wissenschaft(en), die 
zur Einsicht in jene Denkbedingungen befähigt ist, auf denen die Spezialwissenschaften aufruhen, ohne sie noch selbst ausweisen zu können (bei Platon etwa die Ideen oder bei Kant die transzendental reinen Formen von Sinnlichkeit und Verstand). Demgegenüber könnte man mit Blick auf das 19. Jahrhundert von einer Art „Einziehung“ der Philosophie in die Funktionen einer positiven Wissenschaftstheorie sprechen: Anstatt - wie es bei Abel Rey heißt: „von außen und von oben herab“ (Rey 1908: 4) - zu reflektieren, was den einzelnen wissenschaftlichen Rationalitäten (die qua Gegenstand und/oder Methode konsequent begrenzt sind) vermeintlich vorausliegt, rückt die Philosophie, was das 19. Jahrhundert betrifft, zu den empirischen Wissenschaften tendenziell in ein Verhältnis der Immanenz. Sie bildet fortan eher einen kritischen Appendix zu der von den Einzelwissenschaften geleisteten Produktion positiver Erkenntnisse und wird darin, wie sich etwa mit Blick auf Marx zeigen lässt, „praxisnäher“. Eine „Philosophie [oder Theorie] der Wissenschaften" im Sinne Whewells scheint demnach nicht so sehr eine Philosophie über die Wissenschaften, sondern eher eine Philosophie aus den Wissenschaften, eine an die Wissenschaften angelehnte Philosophie zu konstituieren.

Vor allem aus dieser Krise der Philosophie heraus erklärt sich drittens, dass sich Wissenschaftstheorie und Wissenschaftsgeschichte ungeachtet der fließenden Übergänge, die sie zueinander unterhalten, nach und nach als zwei voneinander unabhängige Disziplinen institutionalisiert haben. So könnte man etwa das Projekt einer philosophy of science (nach dem Modell der in der angloamerikanischen Tradition dominanten analytischen Beschäftigung mit den Gültigkeitsbedingungen, den erkenntnistheoretischen Grundlagen und den logischen Implikationen wissenschaftlichen Wissens), den Logischen Empirismus oder den Kritischen Rationalismus dem wissenschaftstheoretischen Arm jenes neuen Nachdenkens über den Wissenschaftsbegriff zurechnen, das im 19. Jahrhundert anhebt. Demgegenüber ließen sich die sogenannte new philosophy of science (Kuhn, Feyerabend, Lakatós) sowie die Historische Epistemologie französischer Provenienz dem prononciert wissenschaftsgeschichtlichen Arm dieser modernen Reflexion zuordnen. Auch ein bestimmter Strang der Phänomenologie, in der Nachfolge von Husserls Krisis der europäischen Wissenschaften und die transzendentale Phänomenologie, ließe sich auf dieser Seite des Bildes unterbringen. Zweifellos existieren vielfältige Mischungen: Gleichwohl kann ein wissenschaftstheoretisches Projekt (beispielsweise Poppers Kritischer Rationalismus) ebenso ohne jegliches Interesse an der Wissenschaftsgeschichte auskommen wie es umgekehrt eine Wissenschaftsgeschichte ohne Aufmerksamkeit für die internen erkenntnistheoretischen Probleme wissenschaftlichen Wissens gibt. 


\section{Zum systematischen Profil der Historischen Epistemologie und zu ihrer deutschsprachigen Rezeption}

Die bereits angesprochene Tradition der Historischen Epistemologie (épistémologie historique) nimmt vor dem Hintergrund der eben resümierten diskursiven Verschiebungen des Wissenschaftsbegriffs noch einmal eine Sonderstellung ein. Der systematisch innovative Impuls, der gerade von dieser Tradition ausging, bestand in einer doppelten Annäherung, nämlich an die je spezifischen Begriffe, in denen wissenschaftliche Diskurse die ihnen jeweils korrelativen Gegenstände objektivieren sowie, im Zusammenspiel mit ihnen, an die materialen Techniken und Praktiken, die Apparaturen und Experimente, die mit diesen Konzeptualisierungen intrinsisch verwoben sind. Obwohl es im Ganzen angemessen erscheint, die Historische Epistemologie als eine französische Affäre wahrzunehmen (weswegen sie gelegentlich auch als „französische Epistemologie“ betitelt wird), fällt seit etwa einem Jahrzehnt in der deutschsprachigen philosophischen Diskussion eine verstärkte und geschärfte Hinwendung zu dieser Denkrichtung auf. Diese neue Aufgeschlossenheit hat, während die traditionelle Rezeptionslage eher von skeptischen bis tendenziös gefärbten Kenntnisnahmen geprägt war, einige Übersetzungen zuvor nicht auf Deutsch vorliegender Grundtexte der Historischen Epistemologie generiert. Eine einschlägige, knappe Auswahl dieser Übersetzungen soll im Folgenden synoptisch besprochen werden: die Abhandlung Über Logik und Theorie der Wissenschaft von Jean Cavaillès, die Anthologie Die Erkenntnis des Lebens von Georges Canguilhem sowie Alexandre Koyrés Studie Paracelsus. Ergänzt wird dieser Textkorpus durch die erste englischsprachige Übertragung von Gaston Bachelards L'intuition de l'instant.

In historischer Hinsicht bezieht sich das Etikett „Historische Epistemologie“ zunächst auf jene Schule innerhalb der französischen Philosophie des 20. Jahrhunderts, die um Autoren wie Gaston Bachelard, Jean Cavaillès, Georges Canguilhem, Michel Foucault oder auch Alexandre Koyré gravitiert. Vor allem aber wäre diesem Bild, gerade um den institutionengeschichtlichen Hintergrund zu konkretisieren, der Name von Abel Rey (1873-1940) hinzuzufügen, der im Jahr 1932 das der Sorbonne unterstellte Institut d'histoire des sciences et des techniques gründete, dessen Leitung ab 1940 Gaston Bachelard als Nachfolger Reys sowie ab 1955 Georges Canguilhem als Nachfolger Bachelards innehatten. ${ }^{1}$ In der Tat lässt sich der Ausdruck „historische Epistemologie“, wie Jean-François Braunstein (Braunstein 2012) vergegenwärtigt hat, auf Reys Einleitung zu seiner Dissertationsschrift Die Theorie der Physik bei den modernen Physikern ([1907] dt. 1908) zurückdatieren. Gegenüber der philosophisch tradierten Bestimmung von Epistemologie als einer „transzendentalen Untersuchung [...] über die Prinzipien und 
allgemeinen Bedingungen der Wissenschaften“ (Rey 1908: 12), so Rey, sei eine "positivistische Wendung“ dieses Begriffs dahin gehend geboten, dass er fortan „den Inbegriff der zur Begründung eines exakten Gesamtbildes der verschiedenen Wissenschaften notwendigen historischen Betrachtungen, eine positive Wissenschaft der Wissenschaft“ denotieren könne. „[D]ie historische Untersuchung des allgemeinen Geistes jeder Wissenschaft wäre dann", so Reys Schlussfolgerung, „eine der großen Sektionen dieser historischen Epistemologie“ (ebd.). Wie adäquat es ist, Rey nicht nur nominell, sondern auch der Sache nach als Begründer des Paradigmas der Historischen Epistemologie zu lesen, demonstriert auch seine Aufforderung an eine moderne Wissenschaftsphilosophie, „auf dem Gebiete der Erkenntnis ihre nützliche und notwendige Aufgabe [wahrzunehmen], indem sie die Reflexionen der Forscher über ihre Methoden von einem synthetisch-kritischen Gesichtspunkt aus aufgreift und zu den Merkmalen, Bedingungen, und Prinzipien des wissenschaftlichen Geistes zurückgeht" (ebd.: 4).

Hier klingen zwei charakteristische Gesten jenes Projekts an, das später auch bei Bachelard, Canguilhem und benachbarten Autoren unter dem Titel „Historische Epistemologie“ firmieren wird: Nämlich zum einen eine „synthetisch-kritische" Anknüpfung an die von den Wissenschaftlern selbst artikulierten methodologischen Reflexionen, die sich in genau dieser Hinsicht als eine gleichsam immanente Anknüpfung pointiert; und zum anderen eine geschichtliche Fokussierung, die den "wissenschaftlichen Geist" von den je konkreten, stets präzise konstellierten „Merkmalen, Bedingungen, und Prinzipien" her erfasst, aus denen heraus er seinen eigenen Diskurs und seine Gegenstände zu einem gegebenen Zeitpunkt konstituiert. Dieser immanent aus den Fragestellungen der Wissenschaftler selbst ansetzende Rekurs auf die historisch bestimmten Begriffe, aber auch auf die Techniken und Praktiken, „mit denen Dingen zu Objekten des Wissens gemacht werden“" (Rheinberger 2007: 11), stellt tatsächlich, angefangen bei Abel Rey, die wesentliche Signatur der Historischen Epistemologie dar, die sie gleichzeitig von den empirischen Wissenschaften (die sie keineswegs unkritisch redupliziert) wie von der Philosophie (der sie sich mitnichten subsumieren lässt) unterscheidet. Georges Canguilhem sollte in den 1960er Jahren diese definitorischen Abgrenzungen Reys in seine eigene Bestimmung von einer Historischen Epistemologie aufnehmen, wonach „eine Theorie der Erkenntnis ohne Bezug auf die Epistemologie ein Meditieren ins Leere wäre und [...] die Epistemologie ohne Bezug auf die Geschichte der Wissenschaften eine völlig überflüssige Verdoppelung der Wissenschaft [...], über die sie etwas auszusagen beansprucht" (Canguilhem 1979: 24; vgl. auch Schmidgen 2014). So aufgefasst, bezieht die Historische Epistemologie ihre kritische Kraft aus dem Umstand, dass sie folgende Spannung gerade zulässt, anstatt sie durch falsche Gegensätze vorschnell zu entschärfen: Während sie in ihrer Rekonstruktion der inneren Normativität und der 
Autokorrekturprozesse der Wissenschaften ein gleichsam positivistisches Moment impliziert, gewinnt sie durch das Insistieren auf der Geschichtlichkeit wissenschaftlichen Wissens zugleich eine - mit Foucault, aber schon mit Abel Rey könnte man sagen: ,archäologische“ - Distanz zu den positivistischen Zügen der wissenschaftlichen Rationalität.

Peter Schöttler hat konzise die wissenschaftspolitischen Blockaden, die innerhalb des nach 1945 bis in die 1990er Jahre hinein ohnehin eher schleppenden, weitgehend in Polemik erstarrten deutsch-französischen Dialogs auch einer aufgeschlossenen Wahrnehmung der Historischen Epistemologie hierzulande entgegengewirkt haben, benannt (Schöttler 2012). Als symptomatisch kann in diesem Kontext etwa der von Schöttler (ebd.: 43) hervorgehobene Umstand gelten, dass die frühen deutschen (Suhrkamp)Übersetzungen der Bücher Althussers und Foucaults (Pour Marx, Folie et déraison, Les mots et les choses) den französischen Ausdruck „épistémologie“ uneinheitlich, nämlich wahlweise mit „Erkenntnistheorie“ oder „Wissenschaftstheorie" wiedergeben: Demgegenüber denke man an die eben erinnerten begrifflichen Abgrenzungen bei Canguilhem.

Weiten Kreisen der gesellschaftstheoretisch orientierten politischen Philosophie und Sozialwissenschaften in der Bundesrepublik hätte der methodische Rahmen Bachelards und Canguilhems (wäre es überhaupt, was nicht geschehen ist, zu einer expliziten Rezeption dieser Autoren gekommen) wohl nur als soziologisch naiv erscheinen können - als ein positivistisches Missverständnis, das nach dem Modell der von Adorno, Horkheimer und Habermas reklamierten Trennung zwischen gesellschaftlicher Totalität und wissenschaftlichem Faktum zu entkräften wäre. ${ }^{2}$ Zugleich stellt die Historische Epistemologie, zumindest in der von Bachelard elaborierten und durch Canguilhem weitergeführten Variante, eine direkte Gegenthese zur phänomenologischen Wissenschaftskritik in den Bahnen von Husserls Krisis der europäischen Wissenschaften dar. Zwar akzeptieren Cavaillès, Bachelard und Canguilhem tendenziell Husserls Analyse, wonach wissenschaftliche Rationalitäten in konkreten historischen Verhältnissen "sedimentiert" seien (dazu Hyder 2003, insbesondere 113). Aber diese Ablagerungen verweisen weder auf einen Ursprung in den konkreten Intentionalitäten der spontan-praktischen „Lebenswelt" noch kann es angehen, die Multiplizität und Regionalisierung der modernen Wissenschaften im Namen einer vermeintlich irreduziblen und einheitlichen ratio, nämlich der phänomenologischen Evidenz der Bewusstseinserlebnisse, $\mathrm{zu}$ kritisieren und begrenzen $\mathrm{zu}$ wollen (siehe Balke 1993). Vielmehr setzen die Autoren der Historischen Epistemologie den „epistemologischen Bruch“ wissenschaftlicher Diskursivität mit der phänomenologischen Anschauung als konstitutiv an.

Last but not least tritt die Historische Epistemologie - zumindest auf den ersten Blick - auch noch in eine diametrale Gegenstellung zu den Positionen der Hermeneutik (Gadamer, auch Habermas) und der Philosophischen 
Anthropologie (Scheler, Plessner, Gehlen), die gleichsam in zweiter Reihe, hinter den bereits erwähnten Modellen der Kritischen Theorie und der Phänomenologie die Gemengelage der bundesrepublikanischen Philosophie nach 1945 mitbestimmten. Dies gilt insbesondere, wenn man Foucaults Einschätzung zu Grunde legt, dass die Standpunkte von Cavaillès, Bachelard, Canguilhem und Koyré auf der Achse einer „Philosophie des Wissens, der Rationalität und des Begriffes“ angesiedelt seien, welche die Linie einer „Philosophie der Erfahrung, des Sinnes, des Subjekts“ (Foucault 1988) Punkt für Punkt konterkariere (vgl. dazu Cassou-Noguès and Gillot 2009).

\section{Von der Philosophie des Bewusstseins zur Dialektik wissenschaftlicher Notwendigkeit: Jean Cavaillès}

In mancher Hinsicht kann man Über Logik und Theorie der Wissenschaft von Jean Cavaillès (Cavaillès 2009) als paradigmatische Begründung jener „französischen Epistemologie“ lesen, deren Rezeption in Deutschland aus den eben dargelegten Gründen traditionell unter einem so ungünstigen Stern stand. Dass die 2011 im Diaphanes Verlag herausgebrachte deutsche Fassung dieses Haupttextes von Cavaillès die überhaupt erste Übersetzung einer wissenschaftlichen Schrift dieses Autors in Deutsche darstellt, grenzt an ein Wunder, wenn man bedenkt, dass die intellektuelle Leistung und das biographische Schicksal von Cavaillès in seiner französischen Heimat Gegenstände einer Verehrung sind, die längst mythisch überhöhende Züge angenommen hat. Sein unerschrockenes Engagement in der französischen résistance, das er, nachdem er zuvor bereits mehrfach verhaftet worden war, im Jahr 1944 mit dem Leben bezahlte, taucht die intellektuellen Hinterlassenschaften von Cavaillès in Philosophie und Mathematiktheorie bis heute in einen alles überstrahlenden Nimbus. Sowohl an der École normale supérieure als auch an der Sorbonne gibt es einen "Salle Cavaillès“, so dass sich Cavaillès an diesen für die französische Kultur symbolisch so aufgeladenen Orten neben Namensgebern wie Descartes oder Malebranche einreiht.

Über Logik und Theorie der Wissenschaft ist ein Text, der eine merkwürdige Ambivalenz zwischen seiner eigenen Form und seinem philosophisch-wissenschaftstheoretischen Thema in sich trägt. Wie Georges Canguilhem und Charles Ehresmann in ihrer Vorbemerkung zu der von ihnen 1946 besorgten Erstausgabe dieses Buches mitteilen, brachte Cavaillès seine Abhandlung 1942 während der Haft in einem südfranzösischen Gefängnis zu Papier. Dank der heimlichen Duldung des zuständigen Militärkommandanten, dem Cavaillès bekannt war, gelang es ihm, bei der Niederschrift auf Quellenliteratur zurückgreifen, die ihm Freunde und Kollegen zuspielten, wann 
immer sie ihn in der Haft besuchten. Das Typoskript, das unter diesen außergewöhnlich prekären Bedingungen entstand, stellt die Quintessenz einer als umfangreicheres Projekt angelegten Untersuchung dar, zu der Cavaillès bereits vor seiner Festnahme Manuskripte verfasst hatte, von denen sich einige verstreut nach seinem Tode, etwa im Besitz seiner Schwester oder in seiner zurückgelassenen Pariser Wohnung, fanden. Insofern hat die veröffentlichte Version von Über Logik und Theorie der Wissenschaft unvermeidlich den Status eines Fragments, dem man fortlaufend anmerkt, dass es aus verlorenen Vorarbeiten herausdestilliert worden ist. Doch dieser fragmentarische und gleichsam ex post aus der Erinnerung an eine verlorene Vorgeschichte $\mathrm{zu}$ neuer Einheit montierte Text zeichnet sich zugleich durch eine luzide Strenge der Gedankenführung aus. Trotz dreier durch römische Zahlen markierter Unterkapitel (die allerdings durch keine erläuternden Zwischenüberschriften betitelt sind) liest sich die Abhandlung wie ein in einem Zug gefertigter Monolith. Weil ausführlichere Herleitungen der Problemstellung sowie der Verbindungen zwischen den von Cavaillès kommentierten Positionen fehlen, erhält die Darstellung einen systematisch-konzentrierten, geradezu rigorosen Charakter.

Im ersten Unterkapitel seiner Schrift exponiert Cavaillès eine Zweideutigkeit innerhalb der Kantschen Wissenschaftssystematik, die dem Zusammenhang zwischen dem "Logik-Teil" der Kritik der reinen Vernunft und Kants Vorlesungen über Logik entspringt. Letztere (im Folgenden mit Logik abgekürzt) bezögen sich auf das, was Kant im Sinne der Tradition als „formale Logik" bestimmt, während die Kritik der reinen Vernunft eine „transzendentale Logik“ grundlege, die allerdings auf dem Modell der als „fix und fertig" (Cavaillès 2011: 27) übernommenen formalen Logik aufruhe. Die nach Begriffs-, Urteils- und Schlusslehre aufgegliederte formale (oder auch „reine“, mit keinerlei empirischen Prinzipien durchsetzte) Logik leiste bereits eine volle Begründung einer apriorischen Wissenschaft von den Verstandesregeln ${ }^{3}-$ und zwar, und dies ist die Pointe bei Cavaillès, ohne Rekurs auf eine transzendentale Synthesis des Verstandes. Kants Transzendentalisierung der formalen Logik im Rahmen einer „Philosophie des Bewusstseins“ substituiert eine „auf einer geringeren Abstraktionsstufe“ (ebd.) bereits geleistete Synthesis, nämlich die in der formalen Logik implizierte logische Handlung der Bildung eines empirischen Begriffs im Ausgang von einer gegebenen Vorstellung. Die transzendentale Synthesis des Verstandes im Sinne der Kritik der reinen Vernunft erweist sich für Cavaillès als supplementär im Verhältnis zu jener empirischen Synthesis, „wodurch [gemäß der Logik Kants, Anmerkung des Autors] in Begriffen, vermittelst der analytischen Einheit, die logische Form eines Urteils zustande" komme (Kant 1922: 98/B 105). Dann aber hat man es, so Cavaillès, bei Kant mit einer Depotenzierung zu tun, die dasjenige, was in den Grenzen der formalen Logik die (empirische) Synthesis stiftet, auf 
das bloß analytische Äquivalent eines Verstandes herabstuft, der die rein synthetische Einheit des Mannigfaltigen in der Anschauung konstituiert. ${ }^{4}$

„In einer Philosophie des Bewusstseins kann die Logik nur eine transzendentale sein" (Cavaillès 2011: 27): Dies ist der Nukleus der Kritik von Cavaillès an Kant. Unterhalb der in die Immanenz des Bewusstseins (in die transzendentale Einheit der Apperzeption) verlagerten Synthesis habe Kant eine empirische Synthesis des Begriffs vollzogen, die seiner transzendentalen Wende ebenso vorausliege wie sie durch sie verdunkelt worden sei. Für Cavaillès schlägt diese Inkonsistenz gerade auch auf Kants schwankende Bestimmung der Rolle der Mathematik durch, die, Kants Definitionen in der Logik zufolge, in ihrem konstitutiven Bezug zur Anschauung noch als Organon jeglicher Wissenschaft überhaupt rangiert, während die Kritik der reinen Vernunft eine außermathematische Grundlegung der reinen Formen der Sinnlichkeit und des Verstands (wie Cavaillès am Beispiel der Kategorie der Kausalität erläutert) unternimmt. Ausgehend von dieser begründungstheoretischen Aporie, die er am Übergang von der formalen zur transzendentalen Logik bei Kant aufdeckt, legt Cavaillès nun zwei Pisten frei, die den weiteren Gang der philosophischen Epistemologie der Mathematik geprägt hätten: Auf der einen Seite die intuitionistische Fundierung der Mathematik bei Luitzen Egbertus Jan Brouwer (sowie bei Léon Brunschvicg, dessen Ansatz Cavaillès, wie denjenigen Brouwers, interessanter Weise als eine „epistemologische [...] Immanenzphilosophie" kennzeichnet; siehe ebd.: 32), auf der anderen Seite die formalistische Tradition von Bernard Bolzano über David Hilbert bis (mutatis mutandis) zu Husserl.

Konsequent also bildet „die Spannung zwischen mathematischer Erkenntnis in Verbindung mit Bewusstsein(-sakten) und der Wissenschaft als [einem] autonomen, vom Beweis regierten System" (Heinzmann 2009) den Schlüssel zur kapitalen These von Über Logik und Theorie der Wissenschaft. In Anspielung auf Hermann Weyl umreißt Cavaillès bereits an einem früheren Punkt seiner Argumentation eine Vorstellung von „Mathematik [als] ein[em] autonome[n] Werden, »mehr ein Tun als eine Lehre«, von dem sich keine Ausgangsdefinition geben lässt, dessen Momente aber in ihrem notwendigen Zusammenhang ein ursprüngliches Wesen erkennen lassen“ (Cavaillès 2011: 33). Man kann die Position von Cavaillès damit als Versuch der strukturalen Konkretisierung jener empirisch-gegenständlichen Synthesen charakterisieren, die Kant durch die ursprünglich-synthetische Einheit der Apperzeption supplementiert hatte; sein Interesse gilt jener „erzeugenden Bewegung“ (ebd.: 44), die dem „vom Beweis regierten System“ der Mathematik selbst inhäriert. Mit Bolzano spricht Cavaillès von einem „Notwendigkeitscharakter der Wissenschaft" (ebd.: 37), der - so sein eigener Akzent gegenüber dem mathematischen Strukturalismus Bolzanos - im Lichte der ihm eigenen generativen, unvorwegnehmbaren Dynamik zu erfassen wäre. Überhaupt sind es die grundlagentheoretischen Impulse Bolzanos für die Elaborierung der 
Analysis im 19. Jahrhundert („Bolzanofunktion“; Beweis des Zwischenwertsatzes), an denen Cavaillès die prononciert moderne Konfiguration wissenschaftlichen Wissens im Sinne „eine $[\mathrm{r}]$ auf sich selbst eingeschränkte[n] Dynamik“ (ebd.: 41), die sich rein intern, „durch spontane Hervorbringung intelligibler Elemente“ (ebd.) selbst trage, weiter erläutert. Zwar stelle Bolzanos Projekt im Ganzen eine „Bereicherung der leibnizianischen Linie“ (ebd.: 39) in der Auseinandersetzung um die Ermöglichung einer mathesis universalis dar, doch während die Mathematik innerhalb der Grundlegung des Infinitesimalkalküls bei Leibniz noch eine intermediäre Stellung „zwischen dem menschlichen Geist und dem Sein an sich“ (ebd.) einnehme, habe Bolzano die Leibniz'sche Maxime „Das Notwendige ist das Bewiesene" (ebd.) dahin gehend radikalisiert, dass er die Generierung des Beweises als inneres und einziges Prinzip des mathematischen Diskurses angesetzt habe.

Es ist markant und zugleich befremdlich, dass Cavaillès die in sich geschlossene Operation des mathematischen Beweises im Sinne einer „Offenlegung, die vom Offengelegten nicht unterschieden, in seiner Bewegung präsent ist", keineswegs als eine statisch-analytische Relation interpretiert, sondern als ein Werden, das nie „zum Stillstand“ komme, sich vielmehr „erweitere" und „verzweige“ (ebd.: 43) geleisteten Beweis ergebende Geltungsbereich intelligibler Größen oder Relationen eo ipso zum Gegenstand neuer Theoreme werde, die durch ihn allererst hervorgebracht würden. Aber nicht genug damit, dass Bolzano, in der Lektüre von Cavaillès, durch die Engführung der mathematischen Bewegung auf die Struktur des Beweises den Diskurs des wissenschaftlichen Wissens als einen sich selbst differenzierenden Gang, oder eher: eine sich selbst differenzierende Immanenz einsichtig gemacht habe. Bemerkenswert sei vielmehr, dass Bolzanos Standpunkt eine „Wissenschaftslehre“ (so ja auch der Titel seines Hauptwerks) fundiere, deren „Aussagen [...] nicht konstitutiv für einen bestimmten theoretischen Entwicklungsgang“ seien, sondern auf eine „Wissenschaft der Wissenschaft" (ebd.: 42) zielten: Insofern handle es sich bei der Explizierung der prinzipiierenden Rolle des Beweises im mathematischen Diskurs um die Angabe des „Apriori“ oder, wie die aufschlussreiche Wendung bei Cavaillès lautet, der „Seele der Wissenschaft, die keine äußeren Voraussetzungen hat, jedoch ihrerseits der Wissenschaft bedarf" (ebd.: 44).

Während Unterabschnitt II von Über Logik und Theorie der Wissenschaft die damit etablierten Grundpfeiler einer wissenschaftlichen Epistemologie in der Diskussion mit dem Logischen Empirismus (vor allem Carnaps) präzisiert, ist Unterabschnitt III auf die Zurückweisung von Husserls apophantischer Überführung des „Problem[s] der Logik [...] in das der transzendentalen Konstitution von Gegenständlichkeiten“ (ebd.: 82) konzentriert. Zwar schließe Husserls Projekt einer transzendentalen Logik nicht mehr, wie Kants gleichnamiges Unternehmen, am Korpus der formalen Logik im Sinne der 
historischen Schultradition an, doch dürfte nach dem bisher Resümierten schon greifbar sein, dass die von Cavaillès reklamierte Metatheorie der Mathematik gerade gegen den phänomenologischen Begründungsanspruch Husserls anschreibt. Zweifellos kann die Infragestellung des Husserlschen Programms, zu der Cavaillès hier gelangt, als die unmittelbar wirkungsträchtigste Erbschaft seines Buches angesehen werden. Sie inspirierte Bachelard, Canguilhem und Foucault in ihren Husserl-Kritiken und schlug damit auch stark in Zusammenhängen außerhalb der von ihm selbst gestellten Fragen nach einer Begründungstheorie der Mathematik ein. Sofern Husserls "Standpunkt“ (ebd.: 89) konsequent der „einer Bewusstseinsphilosophie“ (ebd.) bleibe, sei kaum auszumachen, wie die gleichwohl von Husserl beanspruchte Konzeption einer umfassenden formalen Logik abgesichert werden könne. An diesem Punkt wird der Parallelismus, den Cavaillès zwischen Kant und Husserl stiftet, flagrant: Auch Husserl oszilliert zwischen einer immanenztheoretischen Fassung der formalen Logik (wonach implizit den rein konzeptuellen Strukturen selbst bereits ein Moment des Synthetischen zuerkannt wird) und einer Philosophie des transzendentalen Ursprungs jeglicher (d.h. auch logischer) Geltung im Bewusstsein. Gerade auf den letzten Seiten von Über Logik und Theorie der Wissenschaft verdichtet sich noch einmal in nuce der Antagonismus zwischen Cavaillès'Verständnis des Fortschreitens einer wissenschaftlichen Rationalität im Sinne einer „beständige[n] Revision der Inhalte durch Vertiefung und Tilgung" und dem bewusstseinsphilosophischen Paradigma des Transzendentalen, das in Gestalt Husserls Anfang des 20. Jahrhunderts unerwartet zu neuer Stärke erwacht war: „Keine Philosophie des Bewusstseins, nur eine Philosophie des Begriffs kann eine Wissenschaftslehre liefern" (ebd.: 103).

\section{Serien des Instantanen und der Unfall als Prinzip: Gaston Bachelard}

Zweifellos weckt der Versuch von Cavaillès, in die axiomatischen Operationen des mathematischen Diskurses selbst eine Potenz der Synthesis und der Spontaneität einzutragen, manche Rückfrage. So bleibt, um nur einen exemplarischen Punkt zu nennen, unterbeleuchtet, inwiefern sich Cavaillès mit den Mitteln seiner Argumentation die Möglichkeit verschaffen kann, eine im engeren Sinne geschichtliche Perspektive auf das mathematische Wissen einzunehmen: Was mithin nicht recht sichtbar wird, ist der Übergang von seiner Zurückweisung der Bewusstseinsphilosophie im Namen einer Epistemologie der Immanenz zu einer an konkreten epistemologischen Brüchen orientierten Archäologie wissenschaftlicher Formationen und Praktiken eine Unklarheit, die durchaus eng mit dem paradigmatischen Feld, auf dem Cavaillès seinen veränderten Wissenschaftsbegriff ausgestaltet, nämlich der epistemologischen Situation der modernen Mathematik im 19. Jahrhundert, 
verknüpft ist. Unübersehbar ist jedoch die programmatische Dimension seiner Abhandlung, nämlich die Überholung eines kumulativen Modells der Fortschrittlichkeit wissenschaftlichen Wissens durch ein differentielles Modell, das den „Fortschritt" einer Wissenschaft gerade an einer Dynamik der Revision und der Elimination festmacht (vgl. ebd.: 103).

Damit wäre die Brücke zu Gaston Bachelard hergestellt, dessen kompaktes Büchlein L'intuition de l'instant nun erstmals in englischsprachiger Übersetzung vorliegt. Die 2013 bei Northwestern University Press erschienene Ausgabe korrespondiert mit der 1992 in der Édition Stock herausgebrachten Neuauflage, weshalb als Additamente, getreu dem französischen Quelltext, Übersetzungen von Bachelards Aufsatz „Instant poétique et instant métaphysique" von 1939 sowie Auszüge aus Jean Lescures begleitendem Kommentar, der neben Bachelards Essay 1939 im zweiten Heft der Revue Messages (Hefttitel Métaphysique et poésie) veröffentlicht wurde, beigefügt sind. Dass diese Schrift Bachelards nunmehr auch nicht frankophonen Lesern zur Verfügung steht, ist nicht zuletzt deshalb so erfreulich, weil Bachelard hier gewissermaßen jene Konzeption eines zeitlich-geschichtlichen Werdens wissenschaftlicher Rationalitäten entwickelt, die Cavaillès',organisch“-generative Auffassung mathematischer Rationalitätsstrukturen zu prägen scheint, von Cavaillès selbst jedoch nicht systematisch offen gelegt worden ist.

Bachelard entwirft seine Überlegungen zur Temporalität des Wissens in direktem Gegenzug zu Henri Bergsons Philosophie der Dauer [durée], letztere verstanden als die sich durchhaltende Fließbewegung der Zeit, die, Bergson zufolge, im Modus subjektiver Intuition als in sich ungeteilte Totalität zur Erfahrung gelange, weshalb sie im Verhältnis zur gemessenen und objektivierbaren Zeit inkommensurabel bleibe. In dem Maße, in dem Bergson die subjektiv als indivisibel erlebte Dauer als authentische Tiefenstruktur der Zeit gegen die objektivierte „Zeit der Uhren“ ausspiele, leiste er, so Bachelard, einer verzerrend-negativen Auffassung des Instantanen (im Sinne von frz. instant = Augenblick, Zeitpunkt hier und jetzt) Vorschub. Bachelards fokussiertes Interesse gilt von Anfang an diesem Ausschnitt der Zeittheorie Bergsons; der holistischen, als bruchlos vorgestellten Subjektivität der durée stellt Bachelard das Bild einer intern differentiellen und fraktionierten Zeitlichkeit entgegen, die sich dem menschlichen esprit in Form einer immersiven Erfahrung, der Erfahrung seiner Koinzidenz mit der Objektivität des Hier und Jetzt eröffnet. Bergsons Intuitionismus hänge einer Sichtweise nach, die den Geist als kontemplatives Vermögen bestimme und die Bachelard durch eine "actual and active notion of the experience of the instant" (ebd.: 12) konterkariert: „Idleness alone lingers; the act is instantaneous" (ebd.). Es ist interessant zu sehen, dass Bachelard seine These über diese bereits im Ansatz dispersive Struktur geistiger "Vollzüge“ mit einer Einsicht in diejenige Dimension zusammenfließen lässt, die er als die Geschichtlichkeit des Wissens versteht: 
But the philosopher who aims at describing the history of things, of living beings, and of the mind - atom by atom, cell by cell, thought by thought - must come to detach facts from one another. For facts, besides being facts, are acts. And acts, however unfinished or unsuccessful, must necessarily begin in the absolute of a birth. Effective history must be described via beginnings. Following Roupnel, we must create a doctrine of the accident as principle. There is but one general law in truly creative evolution - the law that an accident lies at the roof of every evolutionary attempt (ebd.: 13, Hervorhebungen im Original).

Mit diesem Argument, das Bergsons kontinuistische Charakteristik der Zeit kritisch gegen sich selbst wendet, nämlich gegen ihr zentrales Implikat eines kontemplativen nous, skizziert Bachelard also zugleich Überlegungen zur Rolle von Zufalls- und Unfallsfaktoren als treibenden Kräften der „effective history". ${ }^{6}$ Die Bergson'sche Dauer wird von Bachelard gleichsam in eine Serialität voneinander unableitbarer, singulärer instants zerstückelt; oder besser: in exakter Umkehrung von Bergsons Auffassung, wonach die in analytische Einheiten unterteilte objektive Zeit eine Projektion darstellt, die von dem unendlichen Kontinuum der durée abstrahiert, liest Bachelard nun die Metaphysik der durée als eine Kontinuitätsillusion, die von der radikalen Dispersität der „instants“ absieht. Hervorzuheben ist im Übrigen der Umstand, dass Bachelard einen denkwürdigen Antipoden zu Bergsons spekulativem Kontinuismus ins Feld führt, nämlich den (mit ihm durch die Universität Dijon freundschaftlich verbundenen) Historiker und Romancier Gaston Roupnel, in dessen kosmologischer Untersuchung Silö̈ (1927) er eine Ontologie der Instantiierung und zugleich der Repetition spiritueller Formen vorgezeichnet sah. Aus dem dogmatischen Schlummer des Bergsonismus (Bachelard 2013: 16) aufgerüttelt habe ihn, so schreibt Bachelard, jedoch vollends Einsteins Theorie der invarianten Eigenzeit als Referenzkriterium für das Problem der Zeitdilatation: „The rise of relativity theory thus suddenly brought to ruin all arguments that had relied on external proofs of a unique, overarching duration as a fundamental principle for the ordering of events" (ebd.: 17).

Bachelards Strategie im weiteren Verlauf seines Buches besteht nun darin, Bergsons Phänomenologie intuitiver Selbst- (als Zeit-) Erfahrung von innen her umzuschmieden: Die zueinander unendlich diskontinuierlich gelagerten „instants“ werden psychisch als Intensitäten des Aufmerkens und des Schmerzerlebens erfahren (ebd. 18-21). Bachelard denkt an eine Verklammerung raum-zeitlicher Instantiierungsereignisse mit einer Psychologie der Aufmerksamkeit, der inneren Wahrnehmung und der Selbstapperzeption, die ausgehend von der erstaunlichen und prägnanten These eingeführt wird, dass "matter is the most uniformly realized habit of being, for it is formed at the very level of the succession of instants" (ebd.: 40). Zahllose Passagen aus L'intuition de l'instant lassen, wie die eben zitierte, tatsächlich an eine ungeahnte Nachbarschaft Bachelards beispielsweise $\mathrm{zu}$ der gegenwärtig breit rezipierten process philosophy Alfred N. Whiteheads denken, deren wesentliche 
Ausrichtung nicht zufällig in einer spekulativen Kosmologie liegt. Ebenso greift Bachelards Argumentation immer wieder auf Phänomene der poetischen Einbildungskraft aus, die in eminenter Weise in die Erhellung dessen involviert zu sein scheint, was Bachelard als die singuläre, in sich unendlich nuancierte Erlebnisdichte der „instants“ bezeichnet.

L'intuition de l'instant ist ein Text, der, wie man nun dankenswerter Weise auch anhand einer englischsprachigen Ausgabe nachvollziehen kann, Zeugnis von Bachelards unerhört fruchtbarer doppelter Sensibilität gibt: Zum einen nämlich für die Originalität und die rationale Schärfe wissenschaftlichen Wissens, zum anderen für die zutiefst intuitiven Kräfte des Poetischen, Traumhaften und Phantastischen. Während Bachelard, überblickt man seine späteren Publikationen, in Formen der Durchdringung von Fragen wissenschaftlicher Rationalität mit Themen der poetischen Dichtung "epistemologische Hindernisse“ erblickte, die über eine „Psychoanalyse" des wissenschaftlichen Geistes abzubauen seien, wagt L'intuition de l'instant gerade einen überaus kühnen Vorstoß, der beide Terrains dezidiert zueinander in Beziehung setzt.

\section{Lebendiges Wissen des Lebens: Georges Canguilhem}

Man könnte die besondere Ausprägung, die Georges Canguilhem dem Denkansatz der Historischen Epistemologie verliehen hat, zunächst als Infragestellung jenes Antagonismus betrachten, den Bachelard zwischen der Spontaneität vorwissenschaftlicher Intuitionen und der rigorosen Diskursivität genuin wissenschaftlicher Vernunft (die durch erstere permanent von innen her getrübt werde) beschrieben hatte (vgl. Canguilhem 1994). Wie die seit 2009 im Berliner August Verlag verfügbare deutsche Übersetzung seiner signifikanten, im Original zuerst 1952 herausgebrachten Aufsatzsammlung $\mathrm{La}$ connaissance de la vie (Die Erkenntnis des Lebens) unterstreicht, hielt Canguilhem die Präsenz von bildlichen Vorstellungen inmitten der Genese jener Begriffe, in denen sich wissenschaftliches Wissen konstituiert, keineswegs für reine Hindernisse. Zwar ist von entscheidender Bedeutung, dass den methodischen Mittelpunkt von Canguilhems historisch-epistemologischen Analysen nun gezielt die Begriffe ausmachen, in denen sich ein wissenschaftlicher Diskurs organisiert (dazu programmatisch Canguilhem 1979), doch wie Canguilhem in seiner 2008 auf deutsch erschienenen Studie über Die Herausbildung des Reflexbegriffs im 17. und 18. Jahrhundert erläutert, versteht er unter einem Begriff (concept) eine Einheit, in der sich der Bezug auf einen Gegenstand (der jeweiligen Untersuchung), eine nominelle Bezeichnung und eine Definition kreuzen. Im besonderen Hinblick auf den durch ihn denotierten Gegenstand drücke ein Begriff stets „eine Handlungs- oder 
Urteilsnorm“ (ebd.: 22) aus, weshalb er „in seinem Umfang nicht variieren kann, ohne auch seinen Inhalt zu verändern “ (ebd.). Gerade diese Verankerung begrifflicher Strukturen im Nährboden einer handlungs- und urteilsleitenden Normativität erlaubt es Canguilhem nun, seine Analysen für die eminente Rolle „naiver“ Anschauungen, gleichsam archetypisch ${ }^{7}$ bildhafter Repräsentationen der dann durch Begriffe angezeigten Gegenstände zu öffnen.

Canguilhem hat die von Rey und Bachelard lancierte Tradition der Historischen Epistemologie in Die Erkenntnis des Lebens explizit als Schlüssel zu einer Geschichte der Wissenschaften vom Leben fruchtbar gemacht. Dieser Schritt ist, wie auch Foucault angemerkt hat, von größtem Gewicht, geht es hier doch weniger um eine schlichte Erweiterung des Gesichtskreises der Historischen Epistemologie um einen Bereich neuer Phänomenalitäten, als um eine grundlegende „Umarbeitung“ (Foucault) dieser Disziplin selbst „an mehreren wesentlichen Punkten“ (Foucault 1988: 60). In letzter Instanz verweist Canguilhem nämlich auf einen Faktor in der Formierung des wissenschaftlichen Wissens (hier: vom Leben), für den unter Bachelards Prämissen, was die Ermöglichung eines spezifisch wissenschaftlichen Diskurses betrifft, kein positiver Platz existieren kann. Dieser Faktor ist die Normativität, die von den wissenschaftlichen Gegenständen selbst - also hier von den Entitäten und Prozessen des Lebendigen - in das wissenschaftliche Geschehen eingebracht wird. So lehnt sich Canguilhem einerseits an Bachelards Behauptung an, dass der wissenschaftliche Gegenstand einen „vom methodischen Diskurs konstituiert[en]“ (Canguilhem 1979: 29) Gegenstand darstellt, der nicht auf den „natürlichen, [den] anfänglichen Gegenstand“ (ebd.) zurückführbar ist; andererseits aber entspringen die Unruhe und die permanente Arbeit der Rektifizierung im Inneren der wissenschaftlichen Begriffsbildung doch einer erratischen Dynamik auf der Seite der (mit dem Begriff zu begreifenden) Phänomene selbst. In der Einleitung zu Die Erkenntnis des Lebens, die ihrerseits den Titel Das Denken und das Lebendige trägt, definiert Canguilhem: „Das Leben ist Herausbildung von Formen [formation des formes], die Erkenntnis ist Analyse geformter Materie [analyse des matières informées]" (Canguilhem 2009: 19). Zum einen wird bereits an dieser Bestimmung sichtbar, dass Canguilhem - verglichen mit Bachelard - mit einer philosophisch weitläufigeren Terminologie operiert: Er formuliert eine Einsicht in die „wesentliche“ Verfasstheit von Leben im Allgemeinen, allerdings nicht unter Missachtung, sondern gerade unter Wahrung der Hypothese Bachelards, dass der Gegenstand eines wissenschaftlichen Diskurses nie ein allgemeines Substrat, sondern stets nur eine spezifische, distinkte Entität sein kann (also nie das Leben schlechthin, sondern etwa eine Zelle oder ein Chromosom). Zum anderen liegt der Akzent, im genannten Zitat wie in der gesamten Konzeption Canguilhems, auf der immer schon formierten Gegebenheitsweise des Lebens. Stets geht es um fest umrissene, eingegrenzte Gestalt(ung)en - nicht bloß organische Körper, sondern auch Strukturen - an 
Hand derer wissenschaftliche Prozesse in Kontakt mit dem Leben geraten. In Absetzung von Bergson argumentiert Canguilhem:

Es stimmt nicht, dass die Erkenntnis das Leben zerstört. Vielmehr nimmt sie die Erfahrung auseinander [défait], um daraus, durch die Analyse des Scheiterns, Gründe zur Vorsicht (Weisheit, Wissen usw.) und eventuell Gesetze für den Erfolg zu abstrahieren und somit dem Menschen bei der Neueinrichtung [refaire] dessen zu helfen, was das Leben ohne ihn, in ihm oder außerhalb seiner gemacht hat [fait]. (ebd.: 17).

Ganz analog dem vorigen Zitat mit seiner Opposition zwischen dem Leben als „formation des formes“ und dem Erkennen, das auf „matières informées“ zurückgeworfen sei, fangen auch diese Sätze einen Chiasmus ein. Die Erkenntnistätigkeit des Menschen, inklusive der Arbeit seiner „wissenschaftlichen" Rationalität, ist eingelassen in eine Expressivität des Lebens selbst, die „ohne ihn, in ihm oder außerhalb seiner" wirksam ist. Erkenntnis ist in dieser Hinsicht ein stets analytisches, diskursives Projekt, das neu zu konfigurieren vermag, was, ontologisch gesprochen, auch unabhängig von ihm ist. Für Canguilhem folgt aus diesem Zusammenhang nun aber gerade keine dualistische Ausschlussrelation von Leben und Erkenntnis wie bei Bergson, sondern das methodische Erfordernis, die theoretischen Kodifizierungen, die Instrumente und die technischen Korrelate jener Erkenntnisse, deren korrelativer Gegenstand das Leben ist, historisch $\mathrm{zu}$ rekonstruieren und voneinander $\mathrm{zu}$ unterscheiden: eben eine Historische Epistemologie der Wissenschaften vom Leben.

Insofern liegt die gehaltvolle Pointe der Analysen Canguilhems darin, Wissenschaft und Technik als Ebenen der Reproduktion (nicht der analytischen Verstellung) von Leben aufzufassen, wenn auch in einem Sinne, der das Selbstverständnis der technischen und wissenschaftlichen Vernunft gerade gegen sich selbst umkehrt. Dies zeigt beispielhaft der in Die Erkenntnis des Lebens enthaltene Aufsatz Maschine und Organismus: Das Mechanische ist hier nicht wie bei Descartes die funktionale Ersetzung des Organischen, die einen lückenlos intelligiblen Diskurs über das Wesen des Lebendigen ermöglichen würde; vielmehr ist die Mechanik im Leben angesiedelt, als ein Zusammenhang von intellektuellen und praktischen Aktivitäten, in denen die spezifische Produktivität von Leben arbeitet. Immer schleicht sich darum in den Versuch der mechanischen Nachahmung des Organischen ein Überschuss ein, der nicht mehr innermechanisch explizierbar ist. Was der maschinelle Nachbau eines Organismus nicht einholen könne, so Canguilhem, sei der Sinn, der seiner eigenen Konstruktion zu Grunde liege, das Wozu der Maschine (ebd.: 183-232). Diesen Sinn beziehe sie aus dem, was sie in funktionaler Hinsicht nachahme, aus einer lebendigen Quelle, in deren Fall der Sinn des ganzheitlichen Zusammenhangs der Teile unmittelbar darin liege, das (Über-) Leben des Organismus zu sichern. 
Wenn die fruchtbare Spannung der Historischen Epistemologie als Disziplin, wie anfangs gesagt, darin besteht, eine bestimmte Idee des positiven Fortschritts von Wissenschaft denken zu können und zugleich, wie oben von Rey zitiert, in kritischer Absicht eine Archäologie der „Merkmale, Bedingungen und Prinzipien des wissenschaftlichen Geistes“ zu stiften, dann ist im Falle von Canguilhem klar, dass sich diese beiden Stränge im Begriff des Lebens verflechten.

\section{Dämmerung des unendlichen Universums: Alexandre Koyré}

Thomas Laugstien, der die Übersetzung von Über Logik und Theorie der Wissenschaft für den Diaphanes Verlag besorgt hat, ist auch die ebenfalls bei Diaphanes erschienene deutsche Fassung von Alexandre Koyrés kurzer Studie Paracelsus [Paracelse], die erstmals 1933 in der Revue d'histoire et de philosophie religieuses der Universität Straßburg veröffentlicht wurde, zu verdanken. Nach der 2008 bei Suhrkamp herausgegebenen zweiten Auflage von Koyrés Hauptwerk Von der geschlossenen Welt zum unendlichen Universum wird damit die Präsenz dieses Autors, der gleichsam an den Grenzen der Tradition der Historischen Epistemologie verortet werden könnte (Chimisso 2008: 3), in der deutschen Rezeption weiter abgesichert. Koyrés Passage in die Wissenschaftsgeschichte französischer Prägung ist berühmt und entsprechend oft nacherzählt worden (Zambelli 1999; Schuhmann 1987; Jorland 1994). Nachdem sich der aus Russland gebürtige Sohn eines reichen jüdischen Unternehmers 1908 in Göttingen niedergelassen hatte, um bei Husserl über einige logischen Antinomien der axiomatischen Mengenlehre zu promovieren, kam es in der Zeit um 1913 zu einem Zerwürfnis mit Husserl, dessen Hintergründe in der damaligen Spaltung der phänomenologischen Schule liegen dürften (Zambelli 1999). Nach seiner Beteiligung an den Frontkämpfen des ersten Weltkriegs etablierte sich Koyré in Paris, allerdings nicht als Phänomenologe, sondern durch Monografien über Descartes, Anselm von Canterbury, Jakob Böhme, später zu Kopernikus und vor allem Galilei. Unter dem Einfluss Émile Meyersons nahm Koyrés Denken eine prononciert wissenschaftsgeschichtliche Orientierung an, wobei seine Verwurzelung im methodischen Horizont der Phänomenologie stets spürbar blieb.

Auf den ersten Blick irritiert es, dass ausgerechnet dieser Paracelsus-Text Koyrés seinen Niederschlag in einer deutschen Übersetzung gefunden hat. Immerhin stellt der Artikel eine Auskopplung aus dem 1955 erstveröffentlichten Band Mystiques, spirituels, alchimistes du XVIe siècle allemand dar, der weitere Aufsätze enthält, die in der vorliegenden deutschen Fassung nicht enthalten sind. Vor allem aber ist der werkgeschichtliche Status dieses 
Paracelsus-Porträts, seine Stellung innerhalb der intellektuellen Denkbewegung Koyrés in den 1930er Jahren schwierig zu bestimmen. Klar ist, dass sich dieser Text nicht als bloße Variation der für Koyré so zentralen These zurechtlegen lässt, wonach die neuzeitliche mathesis universalis (Kopernikus, Galilei) die aristotelisch-mittelalterliche Kosmologie mit ihrer Dualität von phyisca coelestis und physica terrestris zerschlagen und durch eine Konzeption des Universums ersetzt habe, „das als offene, unbegrenzt ausgedehnte Gesamtheit existiert, beherrscht und geeint durch die fundamentalen Gesetze, die überall darin gelten" (Koyré 1994: 71). Freilich führt Paracelsus auch auf einen anderen Schauplatz: Nicht in die Zeit der Selbstbegründung der Mathematik als einer universalen Epistemologie der Natur, sondern eben in eine Welt der Renaissance, die eher noch den mystischen Kosmologien des Mittelalters als der Luzidität der neuzeitlichen Ratio nahe gestanden habe, wie Koyré einleitend herausstellt (Koyré 2012: 9 f.).

In seinem Porträt des Naturheilkundlers, Alchemisten, Astrologen und „Naturphilosophen“ Paracelsus scheint es Koyré nun vor allem um jenen eigenartigen Aufbruch, jenes Tauwetter zu gehen, das in den ersten Jahrzehnten des 16. Jahrhunderts die scholastischen Welterklärungssysteme des Mittelalters $\mathrm{zu}$ verflüssigen und eine vitale Freiheit der Spekulation $\mathrm{zu}$ ermöglichen beginnt, die dann grundlegend für die Entwürfe der neuzeitlichen Mathematiken und Physiken werden sollte:

Für Paracalsus, und darin ist er ein Kind seiner Zeit, ist die Natur weder ein System von Gesetzen noch eines von Körpern, die von Gesetzen regiert werden. Die Natur ist die magische Lebenskraft, die unablässig ihre Kinder zeugt, erschafft und zur Welt bringt. [...] Es waren, wie wir meinen, keine spekulativen Schlüsse, die Paracelsus, nicht anders als den Großteil seiner Zeitgenossen, zu seinem magischen Panvitalismus führten. Es war [...] [d]ie Haltung eines Geistes - einer Seele? -, die sich der Welt nicht entgegenstellte, sondern mit ihr zusammenlebte, ihre Verwandtschaft mit ihr verspürte, die sich vor allem als einen Teil der Welt und des Universums verstand und auch dann, wenn sie der Welt gegenübertrat, nicht die vitalen Bindungen vergaß, durch die sie mit ihr zusammenhing (ebd.: $18 \mathrm{f}$.).

Man kommt, wenn man diese Stellen liest, nicht umhin, sich ins Gedächtnis zu rufen, dass dieser Text tatsächlich zuerst im Jahr 1933 erschien. Die anarchisch-vitalistische Atmosphäre, die Koyré für das beginnende 16. Jahrhundert rekonstruiert, scheint auf das irrationalistische, Lebensphilosophie und Geistesmystik verschmelzende Klima in der europäischen Wissenschaft und Literatur der 1920er und 1930er Jahre anzuspielen. Insofern ist auch die Schlusspointe, die Koyré in seiner Abhandlung setzt, wohl nicht als ein bloßer Rückverweis auf die spiritualistische Theologie des 16. Jahrhunderts einzuordnen:

Ganz unmittelbar wirkt die Gnade als göttliche Tinktur auf die Seele ein, indem sie ihre geistigen Kräfte weckt. Und es ist der Geist, der Glaube - die Kraft des Glaubens, die Imagination -, die den Menschen rettet oder verdammt, je nachdem, ob er an Gott glaubt, an den Teufel oder an die Natur. [...] Der Mensch hat 
sein Werk zu vollbringen, so wie Gott das seine vollbringt. Seinen Auftrag zu erfüllen, sich von Gott lieben und erleuchten zu lassen, sich von Christus, vom Heiligen Geist, dem Parakleten leiten zu lassen (ebd.: 73 bzw. 75).

Das Paracelsus-Buch erscheint in erster Linie als Vehikel eines spirituellen Katholizismus. Die Hinwendung zum Glauben erfolgt in einer post-dogmatischen, ebenso obskuren wie luziden Phase der Geschichte, die gleichzeitig eine Periode des Krieges und der täglichen Drohung durch den Tod ist. Zugleich jedoch fällt an diesem Punkt, insofern Koyré hier eine Serie von ausgeprägt auf Einzelpersonen fokussierten wissenschaftsgeschichtlichen beziehungsweise philosophischen (Böhme, Anselm von Canterbury) Monographien fortsetzt, eine Besonderheit von Koyrés Epistemologie auf, nämlich die Sichtbarmachung „epochaler" wissenschaftlicher Ideen ausgehend von dem souveränen Denk- und Lebensweg eines einzelnen, bedeutsamen Autors. Bei Koyré gipfelt die Beschreibung der wissenschaftlichen Entwicklungen einer Zeit ganz deutlich in den theoretischen Gehalten von Wissenschaft (im Unterschied etwa zu ihren „materialistisch“ relevanten Dimensionen, etwa technischen Dispositiven). Nicht zuletzt in diesem Primat der theoria, der auch seinen Paracelsus-Text prägt, kann man getrost eine Resonanz der Husserl'schen Phänomenologie vernehmen.

In seiner eigenen Denkbewegung hat Koyré die Kontinente der Husserl'schen Phänomenologie und der französischen Historischen Epistemologie überbrückt. Die hier rezensierten Übersetzungen lassen hoffen, dass ein solcher Brückenschlag inzwischen auch für eine ganze Generation von an Fragen der Wissenschaft interessierten Philosophen hierzulande möglich ist.

\section{Anmerkungen}

1 Zur Relevanz von Abel Rey als Scharnier zwischen der positivistischen Wissenschaftsphilosophie und dem durch Bachelard und Canguilhem elaborierten Ansatz einer „Historischen Epistemologie" siehe Braunstein 2012: 37.

2 Interessanter und ironischer Weise hallt eine gewisse Resonanz der Positivismuskritik der Kritischen Theorie ausgerechnet bei Bruno Latour nach, wenn er der Tradition um Bachelard und Canguilhem eine Verengung des Verständnisses von Geschichtlichkeit auf das Feld der Wissenschaftsgeschichte anlastet, wodurch ein Dualismus von Wissenschaft und Ideologie perpetuiert werde - während es Latour, gegen die Fortschreibung der neuzeitlichen Dualismen und Asymmetrien, gerade (worin dann freilich eine krasse Differenz zur Kritischen Theorie aufbricht) um die Symmetrisierung des Hybriden geht (siehe Latour 1998: 125 f.). Sowohl gegen die Muster des sogenannten „Positivismusstreits“ als auch gegenüber Latours Darstellung wäre die oben angedeutete Komplexität der Historischen Epistemologie zu verteidigen, die keine naiven Dualismen bedient, sondern in enger Auseinandersetzung mit den Wissenschaften deren positivistisches Moment zugleich zulässt und geschichtlich distanziert.

3 Siehe hierzu übrigens grundlegend Prien (2006).

4 „Anders gesagt, die Einheit des empirischen Begriffs muss der Erfahrung entnommen werden, sie ist Analyse eines Gegebenen. Wenn aber die den Verstand isolierende Abstraktion diesen analytischen Charakter behält, muss sie an der Vorstellung eines 
bestimmenden Gegebenen festhalten. Dann ist nicht mehr ersichtlich, wie man zum synthetischen Stadium zurückkommt: Wenn unsere Interpretation der Bezugnahme auf das Bewusstsein richtig ist, wo die Ausschaltung des Äußerlichen den absoluten Kern zum Vorschein bringt, kann dies nicht derselbe Verstand sein, der auf einer geringeren Abstraktionsstufe synthetisch war und am Ende analytisch wird - genauso wie auch die Analyse nicht Grundlage der Synthese sein kann" (Cavaillès 2011: 26 f., Hervorhebung des Autors]

$5 \mathrm{Zu}$ dieser fruchtbaren, wenngleich in mehrfacher Hinsicht allemal "gewagten“ Überformung eines spinozistischen Monismus durch eine Philosophie synthetischen Werdens bei Cavaillès siehe Peden (2014), der hier den Hintergrund eines epistemologisch entfalteten Spinozismus in der modernen französischen Philosophie zu Grunde legt.

6 Siehe den erhellenden Aufsatz von Wulz (2012) zu Komplementaritäten zwischen diesem Standpunkt Bachelards in L'intuition de l'instant und der Kategorie des Aleatorischen in den späten Texten Louis Althussers.

7 Dass hier nicht an den Jung'schen terminus technicus zu denken ist, sei an dieser Stelle, um möglichen Missverständnissen entgegenzutreten, nur kurz vermerkt.

\section{Literatur}

Bachelard, Gaston 2013. Intuition of the Instant. Evanston/Illinois: Northwestern University Press. Balke, Friedrich 1993. Das Ethos der Epistemologie. In: Gaston Bachelard. Epistemologie. Frankfurt am Main: Fischer Verlag: 235-252.

Braunstein, Jean-François 2012.Historical Epistemology, Old and New. In: Epistemology and History. From Bachelard and Canguilhem to Today's Philosophy of Science [= Preprint 434 of the Max-Planck-Institut für Wissenschaftsgeschichte]: 33-40.

Canguilhem, Georges 1979. Der Gegenstand der Wissenschaftsgeschichte. In: Ders. Wissenschaftsgeschichte und Epistemologie. Gesammelte Aufsätze. Frankfurt am Main: Suhrkamp: 22-37.

Canguilhem, Georges 1994. Dialectique et philosophie du non chez Gaston Bachelard. In: Ders. Etudes d'histoire et de philosophie des sciences. Paris: Vrin: 197-207.

Canguilhem, Georges 2008. Die Herausbildung des Reflexbegriffs im 17. und 18. Jahrhundert. München: Fink.

Canguilhem, Georges 2009. Die Erkenntnis des Lebens. Berlin: August Verlag.

Cassou-Nougès, Pierre und Pascale Gillot 2009. Le concept, le sujet et la science. Cavaillès, Canguilhem, Foucault. Paris: Vrin.

Cavaillès, Jean 2011. Über Logik und Theorie der Wissenschaft. Zürich: Diaphanes.

Chimisso, Cristina 2008: Writing the History of the Mind: Philosophy and Science in France, 1900 to 1960s. Aldershot: Ashgate.

DAlembert, Jean-Baptiste le Rond 1765. Article „Philosophie“. In: Encyclopédie ou Dictionnaire raisonné des sciences, des arts et des métiers. Bd. 12. Paris: Le Breton: 511-515.

Diemer, Alwin 1968. Die Begründung des Wissenschaftscharakters der Wissenschaft im 19. Jahrhundert - Die Wissenschaftstheorie zwischen klassischer und moderner Wissenschaftskonzeption. In: Alwin Diemer (Hg.). Beiträge zur Entwicklung der Wissenschaftstheorie im 19. Jahrhundert. Vorträge und Diskussionen im Dezember 1965 und 1966 in Düsseldorf. Meisenheim am Glan: Hain Verlag: 3-62.

Foucault, Michel 1988. Das Leben: die Erfahrung und die Wissenschaft. In: Marcelo Marques (Hg.). Der Tod des Menschen im Denken des Lebens. Georges Canguilhem über Michel Foucault, Michel Foucault über Georges Canguilhem. Tübingen: Edition Diskord: 52-72.

Heinzmann, Gerhard 2009. Eintrag „Jean Cavaillès“. In: Thomas Bedorf und Kurt Röttgers (Hg.). Die französische Philosophie im 20. Jahrhundert. Darmstadt: Wissenschaftliche Buchgesellschaft: 94-97.

Hyder, David 2003. Foucault, Cavaillès and Husserl on the Historical Epistemology of the Sciences. Perspectives on Science (11) Heft 1: 107-129. 
Jorland, Gérard 1994. Koyré phénoménologue? In Carlo Vinti (Hg.). Alexandre Koyré. L’avventura intellettuale. Napoli: Ed. Scientifiche italiane: 105-126.

Kant, Immanuel 1922. Kritik der reinen Vernunft (= Werke Bd. III). Berlin: Bruno Cassirer.

Koyré, Alexandre 1994. Galilei und die wissenschaftliche Revolution des 17. Jahrhunderts. In: ders. Leonardo, Galilei, Pascal. Die Anfänge der neuzeitlichen Naturwissenschaft. Frankfurt am Main: Fischer Verlag: 70-87.

Koyré, Alexandre 2008. Von der geschlossenen Welt zum unendlichen Universum. Frankfurt am Main: Suhrkamp.

Koyré, Alexandre 2012. Paracelsus. Zürich: Diaphanes.

Latour, Bruno 1998. Wir sind nie modern gewesen. Versuch einer symmetrischen Anthropologie. Frankfurt am Main: Fischer Verlag.

Peden, Knox 2014. Spinoza contra Phenomenology: French rationalism from Cavaillès to Deleuze. Stanford: Stanford University Press.

Prien, Bernd 2006. Kants Logik der Begriffe. Die Begriffslehre der formalen und transzendentalen Logik Kants [= Kantstudien Ergänzungshefte Nr. 150]. Berlin: de Gruyter.

Pulte, Helmut 2004. Eintrag „Wissenschaftstheorie; Wissenschaftsphilosophie“. In: Joachim Ritter, Karlfried Gründer und Gottfried Gabriel (Hg.). Historisches Wörterbuch der Philosophie. Bd. 12. Darmstadt: Wissenschaftliche Buchgesellschaft: 974-982.

Rey, Abel 1908. Die Theorie der Physik bei den modernen Physikern [= Philosophisch-Soziologische Bücherei Bd. XII]. Leipzig: Klinkhardt.

Rheinberger, Hans-Jörg 2007. Historische Epistemologie zur Einführung. Hamburg: Junius.

Schmidgen, Henning 2014. Die Frage nach der Wissenschaft. In: Hans-Jörg Rheinberger (Hg.). Rekurrenzen. Texte zu Althusser. Berlin: Merve: 7-19.

Schöttler, Peter 2012. Sur la réception de l'„épistémologie française“ en Allemagne. In: Epistemology and History. From Bachelard and Canguilhem to Today's Philosophy of Science [= Preprint 434 of the Max-Planck-Institut für Wissenschaftsgeschichte]: 41-46.

Schuhmann, Karl 1987. Koyré et les phénoménologues allemands. In: Pietro Redondi (Hg.). Science: The Renaissance of a History. Proceedings of the International Conference Alexandre Koyré (= History \& Technology 4): 149-167.

Whewell, William [1840] 1967. The Philosophy of the Inductive Sciences. Part 1. London: Frank Cass \& Company Limited.

Wulz, Monika 2012. Intervals, Possibilities, and Encounters. The Trigger of a Ruptured History in Bachelard. In: Epistemology and History. From Bachelard and Canguilhem to Today's Philosophy of Science [= Preprint 434 of the Max-Planck-Institut für Wissenschaftsgeschichte]: 63-75.

Zambelli, Paola 1999. Alexandre Koyré im ,Mekka der Mathematik'. NTM. Zeitschrift für Geschichte der Wissenschaften, Technik und Medizin (7): 208-230.

Thomas Ebke

Institut für Philosophie

Universität Potsdam

Am Neuen Palais 10, Haus 11

14469 Potsdam

Deutschland

E-Mail: ebke@uni-potsdam.de 